\title{
Relaxation time spectrum molecular weight distribution relationships
}

\author{
Christian Friedrich • Richard J. Loy • \\ Robert S. Anderssen
}

Received: 21 May 2008 / Accepted: 23 September 2008 / Published online: 30 October 2008

(C) Springer-Verlag 2008

\begin{abstract}
Single exponential decay $\exp (-t / \tau)$ relationships, which define the molecular weight distribution (MWD) of a polymer as a function of the polymer's relaxation time spectrum (RTS), have been derived by Wu (Polym Eng Sci 28:538-543, 1988) and Thimm et al. (J Rheol 43:1663-1672, 1999). Experimental validation studies with monodisperse polymers, with quite precisely known MWDs, have been used to test their reliability. It has been established that neither formula is always able to accurately recover the MWDs of monodisperse polymers from their experimentally determined RTS. In this paper, different and more general relationships, based on theoretical results of Anderssen and Loy (Bull Aust Math Soc 65:449-460, 2002a) for decays of the form $\exp (-\theta(t) / \tau)$, where the derivative of $\theta(t)$ is a completely monotone function, are derived, analyzed, and applied. It is shown how to transform these general relationships to equivalent single exponential decay relationships for which Laplace transform solutions are derived. In order to illustrate the interrelationship between an RTS and its corresponding MWD, an explicit analytic solution is given.
\end{abstract}

C. Friedrich

FMF, University of Freiburg (im Br.), Freiburg, Germany

R. J. Loy

Mathematical Sciences Institute, Australian National University, Canberra, Australian Capital Territory, 0200, Australia

R. S. Anderssen ( $\square)$

CSIRO Mathematical and Information Sciences, GPO Box 664, Canberra, Australian Capital Territory, 2601, Australia

e-mail: Bob.Anderssen@csiro.au
The paper concludes with a discussion of the rheological implications for the BSW model.

Keywords Relaxation time spectrum •

Molar mass distribution - Laplace transformation .

Monodisperse - Complete monotonicity •

Double reptation $\cdot$ Molecular weight distribution •

BSW spectrum

\section{Introduction}

From an inverse-problems perspective, the relaxation spectra of a given polymer, derived numerically from the stress-strain dynamics of shear and/or elongation experiments, can be viewed as an indirect measurement of the molecular weight distribution (MWD) of that polymer. Independent analytic formulas have been derived that define the MWD of a polymer as a function of its relaxation time spectrum (RTS). Here, such formulas will be referred to as "relaxation time spectrum-molecular weight distribution" (RTSMWD) relationships. Two specific relationships are widely used to recover approximations to the MWD of a polymer from approximate "estimates" of its RTS. One is due to $\mathrm{Wu}$ (1988). It appears to have been the first such relationship to have been derived. A generalization and popular alternative is that due to Thimm et al. (1999).

Various experimental validation studies with monodisperse polymers, with quite precisely known MWDs, have been performed (Maier et al. 1998; Thimm et al. 2000; Friedrich et al. 2008). For a single monodisperse polymer, with its MWD a very close approximation 
to a Dirac delta function with known molecular mass $m_{0}$, the application of neither the Wu nor the Thimm formula is able to accurately reproduce good approximations to the delta function. The approximate MWDs that are obtained tend to be too broad, especially in the direction of the lower molecular weights. In addition, depending on the assumed form for the function that relates relaxation times to molecular weights, the approximate MWDs might not be centered close to $m_{0}$. Interestingly, for quite broad MWDs, these formulas give approximations with much closer agreement.

A possible explanation relates to the derivations used by $\mathrm{Wu}$ (1988) and Thimm et al. (1999) in the formulation of their relationships. In Wu (1988), his basic equation (Eq. 1 below) is derived by assuming that monodisperse components form polydisperse blends through discrete binary chain contacts. A proof that this basic equation has the solution (Eq. 5 below) that he gives is derived in Section Rheological background. Though the Thimm et al. (1999) result is based on an interesting and useful identity, it involves a time dependence that is removed by assuming that the result holds for all time and, in particular, for time zero. Interestingly, both appear to be useful rules of thumb for industrial decision-making purposes (Cocchini and Nobile 2003; Nobile and Cocchini 2000, 2001).

It is the above-mentioned validation experiments that have led to the conclusion that a more rigorous RTS-MWD relationship is required, and one which yields a much better recovery of the delta function of a monodisperse polymer with the bias to lower molecular weights eliminated. Anderssen and Loy (2002a) have theoretically derived a quite general RTS-MWD relationship that is independent of time but limits the mixing rule reptation parameter to be a rational fraction. Here, in this paper, the applicability and utility of this result is investigated from a practical rheological perspective.

In particular, it is shown how to transform RTS-MWD relationships, which involve decays like $\exp (-\theta(t) / \tau(m))$, where the derivative of $\theta(t)$ is a completely monotone function, to single exponential decay $\exp (-t / \tau(m))$ relationships. This is a more general framework than considered by previous authors and allows for more general decay behavior, which is a possibility that has been overlooked until it was suggested by Anderssen and Loy (2002a). It is then shown how the single exponential relationships can be solved using Laplace transforms. A simple explicit and illustrative solution is also derived using the Laplace transform relationships. It allows the relationship between the RTS and the corresponding MWD to be explored analytically.
The paper has been organized in the following manner. Section Rheological background contains a brief discussion about the background to the problem, the derivations of $\mathrm{Wu}$ (1988) and Thimm et al. (1999), and the generalization of models for the relaxation modulus. A brief review of the Anderssen and Loy (2002a) results, adapted for the determination of an MWD from RTS data, is given in Section The general RTS-MWD relationship. A general strategy for solving the quite general RTS-MWD relationship given in Section The general RTS-MWD relationship is also discussed there. The special cases of single and double reptation are the focus of Section Laplace transform and convolutional RTS-MWD relationships, where a Laplace transform and convolutional solution are derived for double reptation. The simplest form of the double reptation RTS-MWD relationship is examined in some detail in Section Application: the double reptation RTS-MWD relationship, and the utility of the resulting explicit formula is examined there. The paper concludes with a discussion of the rheological implications for the BSW model in Section Rheological implications illustrated with the BSW model.

\section{Rheological background}

The RTS $H(\tau)$ is defined to be $H(\tau)=H_{\text {comp }}(\tau) / G_{N}^{0}$, where $H_{\text {comp }}$ denotes the spectrum derived computationally from experimental data (Ferry 1980) and $G_{N}^{0}$ denotes the plateau modulus. The normalization $\int_{0}^{\infty}(H(\tau) / \tau) d \tau=1$ is thereby guaranteed.

The molecular weight counterpart of $H(\tau)$ is given by $h(m)=H(\tau(m))$, where $\tau(m)$ denotes the assumed "relaxation time $\tau$ "-"molecular weight $m$ " function.

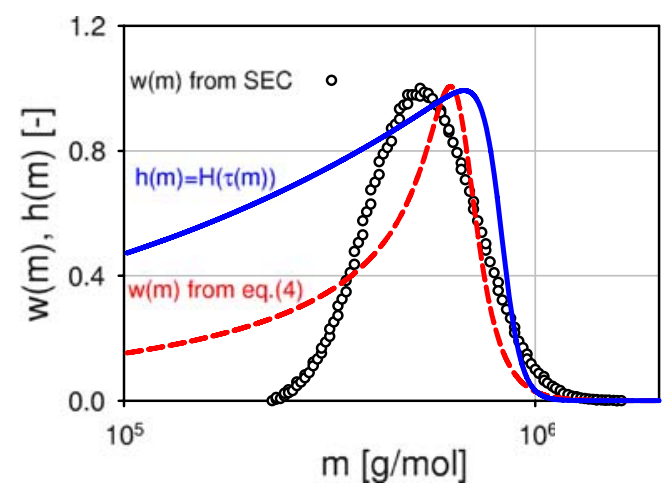

Fig. 1 A comparison of the measured MWD for the polymer PS644 (circles) with the MWD (dashed line) derived from Eq. 4 for the modified BSW spectrum with $\beta=5$ (solid line) of Eq. 43, which was fitted to the relaxation time spectrum data derived experimentally from the measured MWD 
The known poor correspondence between the measured and computed MWDs is illustrated in Fig. 1. As detailed in Section Rheological implications illustrated with the BSW model, a modified BSW model has been fitted to the relaxation spectrum determined for the measured MWD (circles). Though the plot of $h(m)$ (solid line), derived from the fitted BSW model, and the corresponding MWD $w(m)$ (dashed line) have similar qualitative structures, this computed MWD has nothing like the peaked structure of the measured MWD (circles) (details about how the $h(m)$ and $w(m)$ of Fig. 1 have been constructed are given in Section Rheological implications illustrated with the BSW model).

It is likely that such qualitative visual correspondences have been the stimulus for the derivation of the different RTS-MWD relationships that have been published in the rheological literature. In addition, they represent indirect validation for the apparent usefulness of such relationships in rheological applications.

However, as explained below and in the literature (e.g., Nobile and Cocchini 2008), the goal is to obtain, from the $h(m)$ for known (measured) MWDs, predictions of $w(m)$ that agree closely with the known MWDs. Here, in this paper, new RTS-MWD relationships are derived that are more appropriate theoretically and rheologically than the ones published previously.

\section{The Wu (1988) relationship}

By assuming that each component in a blend of $N$ monodisperse species contributes to the relaxation modulus of the resulting polydisperse blend through binary chain contacts, and then going to a continuous limit, Wu (1988) derived the following RTS-MWD integral relationship [which uses a slightly different notation from that of $\mathrm{Wu}(1988)]$ :

$h(m)=2 w(m) \int_{m}^{\infty} \frac{w(\bar{m})}{\bar{m}} d \bar{m}$,

where $h(m)$ denotes the RTS as a function of the molecular weight $m$ and $w(m)$ the volume-fraction differential MWD function. Because of the binary chain assumption, Eq. 1 and its solution can be viewed as RTS-MWD approximate encapsulations of the double reptation process.

$\mathrm{Wu}$ (1988), however, did not give a proof of his solution. It is easily proved in the following manner. On introducing the substitution

$$
R(m)=\int_{m}^{\infty} \frac{w(\bar{m})}{\bar{m}} d \bar{m}
$$

Eq. 1 becomes

$\frac{h(m)}{m}=-2 \frac{d R(m)}{d m} R(m)=-\frac{d[R(m)]^{2}}{d m}$,

which solves to yield the solution given by $\mathrm{Wu}$ (1988)

$w(m)=\frac{h(m)}{2}\left[\int_{m}^{\infty} \frac{h(\bar{m})}{\bar{m}} d \bar{m}\right]^{-1 / 2}$.

Taking into account that it is generally assumed that the relaxation time $\tau$, as a function of $m$, takes the form $\tau(m)=\mathrm{km}^{\alpha}$, Wu's RTS-MWD relationship Eq. 4 becomes

$w(m)=\frac{\alpha^{1 / 2} H(\tau)}{2}\left[\int_{\tau}^{\infty} \frac{H(\bar{\tau})}{\bar{\tau}} d \bar{\tau}\right]^{-1 / 2}$,

$H(\tau)=h(m(\tau))$.

Note The MWD $w(m)$ of Fig. 1 has been determined using Eq. 4 with $h(m)$ derived from the BSW model as discussed in Section Rheological implications illustrated with the BSW model.

The Thimm et al. (1999) relationship

In the formulation of their RTS-MWD relationships, Thimm et al. (1999) utilize the following identity

$$
\begin{aligned}
& -\int_{a}^{\infty} \frac{d}{d x}\left[\int_{x}^{\infty} f(\bar{x}) d \bar{x}\right]^{\gamma} d x=\left[\int_{a}^{\infty} f(\bar{x}) d \bar{x}\right]^{\gamma} \\
& =\gamma \int_{a}^{\infty} f(x)\left[\int_{x}^{\infty} f(\bar{x}) d \bar{x}\right]^{\gamma-1} d x
\end{aligned}
$$

With it, assuming that $\tau(m)=\mathrm{km}^{\alpha}$, they derived a number of quite general results for an arbitrary reptation parameter $\beta$ (their Eq. 12)

$h(m)=\frac{\beta w(m)}{\alpha}\left[\int_{m}^{\infty} \frac{w(\bar{m})}{\bar{m}} d \bar{m}\right]^{\beta-1}$,

and (their Eq. 8)

$w(m)=\left(\frac{\alpha^{1 / \beta}}{\beta}\right) h(m)\left[\int_{m}^{\infty} \frac{h(\bar{m})}{\bar{m}} d \bar{m}\right]^{1 / \beta-1}$.

The similarity between Eq. 5 and the double reptation version of Eq. 8, when $\beta=2$, has led to the assumption that Eq. 8 represents a generalization of and useful alternative to Eq. 5 .

The derivation of Eqs. 7 and 8 is potentially problematic for the following reasons. In Eq. 8, one is working with powers of completely monotone functions that are not necessarily completely monotone (Anderssen and Loy 2002a). In the derivation of both Eqs. 7 and 8, given in Thimm et al. (1999), one obtains a relationship that involves a time dependency, which is removed by 
assuming that it holds in general and in particular for $t=0$. A similar expedient has been utilized implicitly by $\mathrm{Wu}(1988)$ by proposing a discrete binary model for the RTS independent of its relationship to the relaxation modulus.

\section{Modelling the relaxation modulus $G(t)$}

In the rheological literature (Ferry 1980), the relaxation modulus $G(t)$ is usually modelled, using a RTS representation, as

$G(t)=\int_{0}^{\infty} F(t, \tau) \frac{H_{F}(\tau)}{\tau} d \tau$,

where the choice of the kernel $F(t, \tau)$ must be such that $G(t)$ is guaranteed to be a completely monotone function; namely, that $G(t)$ satisfies

$(-1)^{n} \frac{d^{n} G(t)}{d t^{n}} \geq 0, \quad$ for all $n$.

The dependence of the RTS on the choice of $F(t, \tau)$ is made explicit by writing it as $H_{F}(\tau)$. Popular choices for the kernel $F(t, \tau)$ include:

1. The Maxwell-mode model with $F(t, \tau)=F_{\text {Max }}(t, \tau)=$ $\exp (-t / \tau)$. In the literature, it is the $H_{\operatorname{Max}}(\tau)$ that is normally referred to as the RTS.

2. The BSW model (Baumgartel et al. 1990) corresponds to the following special choices for $F(t, \tau)$ and $H_{F}(\tau)$ (Nobile and Cocchini 2008, Eq. 10)

$$
\begin{aligned}
& F(t, \tau)=F_{\mathrm{BSW}}(t, \tau)=\exp (-t / \tau), \\
& H_{F}(\tau)=H_{\mathrm{BSW}}(\tau)=G_{N}^{0} n \frac{\tau^{n+1}}{\lambda^{n}}, \quad \tau<\lambda,
\end{aligned}
$$

zero elsewhere,

where $n$ and $\lambda$ are parameters. In fact, it is a particular case of the Maxwell mode model of 1 .

3. The contour length fluctuation (CLF) model (Doi 1981).

4. Thimm et al. (1999) and Nobile and Cocchini (2008), as well as others, have discussed the use of more general models for $G(t)$. They are less general than the form proposed and analyzed in the sequel in that $F(t, \tau)$ is always assumed to take the form $\exp (-t / \tau(m)) \bar{F}(\tau)$ where $\bar{F}(\tau)$ is a function only of $\tau$. As explained by Nobile and Cocchini (2008), their $h_{\mathrm{BSW}}(\tau)$ has this specialized structure, as does the Rouse model of van Ruymbecke et al. (2002).

All these models for $G(t)$ contain a term of the form $\exp (-t / \tau(\cdot))$, where $\tau(\cdot)$ is independent of $t$. It is the presence of this term that guarantees the complete monotonicity of the relaxation modulus corresponding to these models. However, as pointed out by Anderssen and Loy (2002a), a more general class of completely monotone functions are generated by the kernels

$F(t, \tau)=F_{\theta}(t, \tau)=\exp (-\theta(t) / \tau)$

where the function $\theta(t)$ satisfies the conditions $\theta(0)=0$ and $d \theta(t) / d t$ completely monotone. In physics, and to a lesser extent in rheology (Macdonald 2000; Husain and Anderssen 2005),

$F_{\mathrm{SE}}(t, \tau)=\exp \left(-t^{\alpha} / \tau\right)$

is an important example where $\theta(t)=t^{\alpha}, 0<\alpha<1$, as it is the generator for the stretched exponential (Kohlrausch, Williams-Watts) function $\exp \left(-t^{\alpha}\right)$ (Anderssen et al. 2004). In the sequel, the generalization Eq. 10 for $G(t)$ will play a key role.

The importance of these more general kernels is that they allow for a more complex fading memory than the simple exponential Maxwell model. The advantage of a more general fading memory is the greater flexibility it gives to approximating the experimentally observed reality of polymer dynamics. As highlighted in Eq. 9, the modelling of $G(t)$ is performed as a trade-off between the choice of $F(t, \tau)$ and $H_{F}(\tau)$. Consequently, for a given $G(t)$, by making different choices for $F(t, \tau)$ and then determining the related $H_{F}(\tau)$, a variety of explanations can be constructed for the same polymer dynamics. In the past, there has been a tendency to assume that $F(t, \tau)$ has the simple exponential structure of the Maxwell model and that the experimentally observed polymer dynamics can be explained exclusively in terms of the structure of the related RTS $H_{F}(t, \tau)$. The BSW and CLF models, and the generalization proposed here, allow for the dynamics to be explained in terms of a fading memory representative of the polymer being studied, with the form of the possible choices for the related RTS being constrained accordingly.

\section{The general RTS-MWD relationship}

On the basis of the discussion in Section Modelling the relaxation modulus $G(t)$, it follows that models for the relaxation modulus should allow for the possibility that different choices can be made for the $\theta(t)$ function, in the kernel function of Eq. 10, as the nature of the polymer dynamics changes. This is consistent with the view that, along the length of an individual polymer chain, different dynamics will be occurring depending on the chemical and topological structure of the chain (Milner and McLeish 1998; Garcia-Franco and Mead 1999). In addition, it is assumed that these different dynamics contribute additively to the structure of $G(t)$. 
This is the rationale adopted by (Anderssen and Loy 2002a, b), which we now review using a less general and more appropriate rheological structure and format.

1. Assume that the relaxation modulus $G(t)$ can be modelled as a weighted sum of components $G_{r}(t)$, $r=1,2, \cdots$, corresponding to the various levels $r$ of reptation occurring simultaneously in the dynamics of a given polymer,

$$
\begin{aligned}
\bar{G}_{M}(t) & =\sum_{r=1}^{M} W_{r} G_{r}(t), \quad W_{r} \geq 0, \\
\sum_{r=1}^{M} W_{r} & =1, \quad M<\infty .
\end{aligned}
$$

2. Model the relaxation modulus for each component $G_{r}(t)$ using the generalized RTS $H_{r}(\tau)$ representation of Eq. 10 (as outlined in Section Modelling the relaxation modulus $G(t))$

$$
\begin{aligned}
G_{r}(t) & =\int_{0}^{\infty} \exp \left(-g_{r}(t) / \tau\right) \frac{H_{r}(\tau)}{\tau} d \tau, \\
H_{r}(\tau) & \geq 0, \quad t \geq 0,
\end{aligned}
$$

with $g_{r}(0)=0$ and $d g_{r}(t) / d t$ completely monotone.

3. Assume that the following general mixing rules hold for the relaxation modulii $G_{r}(t)$

$$
\begin{aligned}
& G_{r}(t)=\left[\int_{0}^{\infty} k_{r}(m) \exp \left(-f_{r}(t) / \tau_{r}(m)\right) w(m) d m\right]^{r}, \\
& k_{r}(m) \geq 0, \tau_{r}(m) \geq 0, w(m) \geq 0,
\end{aligned}
$$

with $f_{r}(0)=0$ and $d f_{r}(t) / d t$ completely monotone. Here, $k_{r}(m)$ plays the role of a scaling factor. Normally (traditionally), it takes the form $1 / m$ to give a logarithmic dependence, but this is not necessarily the most appropriate choice. This is a matter that requires further investigation. Except for some short comments below, it is a matter outside the scope of the current paper.

4. Utilize the basic properties of completely monotone functions.

Using the above assumptions, Anderssen and Loy (2002a) established, on equivalencing these two different relationships for $G_{r}(t)$ and ensuring that the complete monotonicity of this equivalencing held in its most general form, that the most general form for $G(t)$ is the summation given in Eq. 11 along with the conditions

$$
\begin{aligned}
& \int_{0}^{\infty} \exp \left(-g_{r}(t) / \tau_{r}(m)\right) \frac{h_{r}(m) \tau_{r}^{\prime}(m)}{\tau_{r}(m)} d m \\
& \quad=\left[\int_{0}^{\infty} k_{r}(m) \exp \left(-f_{r}(t) / \tau_{r}(m)\right) w(m) d m\right]^{r},
\end{aligned}
$$

where $h_{r}(m)=H\left(\tau_{r}(m)\right) / G_{N}^{0}$ and $\tau_{r}^{\prime}(m)=d \tau_{r}(m) / d m$.
Note Because, following $\mathrm{Wu}$ (1988) and Thimm et al. (1999), the goal has been to equate the two different models for $G_{r}(t)$ in order to derive an RTS-MWD relationship, it is necessary to ensure that the MWD representation for $G_{r}(t)$ is also a completely monotone function. This is guaranteed by imposing the conditions on $f_{r}(t)$ given in (3) and by requiring that $r$ be an integer. As explained in Anderssen and Loy (2002a), in general, $r$ cannot be a real number that is not a rational number.

Note In Anderssen and Loy (2002a, b), it was assumed that the MWD in the mixing rules could depend on $r$. This allows for the possibility that only some components of the MWD are related to a specific $G_{r}(t)$. Here, it is assumed that each mixing rule corresponding to each $G_{r}(t)$ sees the same MWD $w(m)$, with the other terms in the integrand of the mixing rule identifying the nature of the dynamics involved.

Note In the above deliberations, it is assumed that $r$ is an integer. More generally, it could be a rational fraction; e.g., $r=r_{n} / r_{d}$, where $r_{n}$ and $r_{d}$ are integers. In such a situation, one would raise the left- and righthand sides to the power of $r_{d}$ thereby obtaining a relationship with the left- and right-hand sides raised to integer powers. The deliberations given below then apply naturally to this more general situation.

As a consequence of the above deliberations, one obtains the following conclusions:

(a) A mixing rule can have a fading memory $g_{r}(t)$ different from the fading memory $f_{r}(t)$ for its RTS counterpart. Currently, the extent to which this might occur appears to be an open question since, currently in the rheological literature, it is usually assumed that $g_{r}(t)=f_{r}(t)=t$. A rationale for there being a difference is that one is associated with a mass relaxation process while the other is associated with a time-dependent relaxation.

(b) In the recovery of the MWD $w(m)$ from a measured relaxation modulus $G(t)$, the initial step must be the decomposition of $G(t)$ into its different components. As explained in Thimm et al. (2000), this must be based on the rheological context in which the $G(t)$ has been measured.

The general strategy for MWD recovery from the general RTS-MWD relationship

As it stands, Eq. 14 can only be solved analytically for $w(m)$ for very special choices of $g_{r}(t), h_{r}(m), \tau(m)$, $k_{r}(m)$, and $f_{r}(t)$, which will not necessarily be representative of realistic rheological situations. However, using 
Bernstein's theorem (Bernstein 1928; Widder 1972), which states that any completely monotone function can be rewritten as the Laplace transform of a positive measure, quite general choices for $g_{r}(t), h_{r}(m)$, $\tau(m), k_{r}(m)$, and $f_{r}(t)$ can be accommodated. On the basis of Bernstein's theorem (Widder 1972, Theorem XIII.4.1a), the integrals on each side of Eq. 14 can be rewritten as

$$
\begin{aligned}
& \int_{0}^{\infty} \exp \left(-g_{r}(t) / \tau_{r}(m)\right) \frac{h_{r}(m) \tau_{r}^{\prime}(m)}{\tau_{r}(m)} d m \\
& =\int_{0}^{\infty} \exp (-t s) \Phi_{r}(s) d s,
\end{aligned}
$$

and

$$
\begin{aligned}
\int_{0}^{\infty} k_{r}(m) \exp \left(-f_{r}(t) / \tau_{r}(m)\right) w(m) d m \\
\quad=\int_{0}^{\infty} \exp (-t s) \Psi_{r}(s) d s .
\end{aligned}
$$

for suitable functions $\Phi_{r}(s)$ and $\Psi_{r}(s)$. A step somewhat analogous to this is made by Nobile and Cocchini (2008) in their Eq. 8, but the connection to complete monotonicity is not made.

On combining these two results, Eq. 14 can be rewritten as

$$
\int_{0}^{\infty} \exp (-t s) \Phi_{r}(s) d s=\left[\int_{0}^{\infty} \exp (-t s) \Psi_{r}(s) d s\right]^{r},
$$

This relationship shows that the solution of the RTSMWD equivalence (Eq. 14) reduces to solving a Laplace transform identity. For example, knowing only either $\Phi_{r}(s)$ or $\Psi_{r}(s)$, the Laplace transform relationship Eq. 15 can be used to recover the other.

As explained in Widder (1972), Theorem XIII.5.2, these two distributions can be reconstructed, using the definition of complete monotonicity, from independently derived values of the derivatives of the left- and right-hand side of Eq. 15.

Introducing the substitution $s=1 / \tau_{r}(m)$ on the right hand sides of the two equations before Eq. 15 then yields

$$
\begin{aligned}
& \int_{0}^{\infty} \exp \left(-g_{r}(t) / \tau_{r}(m)\right) \frac{h_{r}(m) \tau_{r}^{\prime}(m)}{\tau_{r}(m)} d m \\
& =\int_{0}^{\infty} \exp \left(-t / \tau_{r}(m)\right) \bar{\Phi}_{r}(m) \frac{\tau_{r}^{\prime}(m)}{\left(\tau_{r}(m)\right)^{2}} d m,
\end{aligned}
$$

and

$$
\begin{aligned}
& \int_{0}^{\infty} k_{r}(m) \exp \left(-f_{r}(t) / \tau_{r}(m)\right) w(m) d m \\
& =\int_{0}^{\infty} \exp \left(-t / \tau_{r}(m)\right) \bar{\Psi}_{r}(m) \frac{\tau_{r}^{\prime}(m)}{\left(\tau_{r}(m)\right)^{2}} d m .
\end{aligned}
$$

Introducing the substitutions

$\bar{\Phi}_{r}(m)=\frac{\bar{h}_{r}(m) \tau_{r}^{\prime}(m)}{\tau_{r}(m)}, \quad \bar{\Psi}_{r}(m)=k_{r}(m) \bar{w}(m)$,

into these last two equations then yields

$$
\begin{aligned}
& \int_{0}^{\infty} \exp \left(-g_{r}(t) / \tau_{r}(m)\right) \frac{h_{r}(m) \tau_{r}^{\prime}(m)}{\tau_{r}(m)} d m \\
& =\int_{0}^{\infty} \exp \left(-t / \tau_{r}(m)\right) \frac{\bar{h}_{r}(m)\left(\tau_{r}^{\prime}(m)\right)^{2}}{\left(\tau_{r}(m)\right)^{3}} d m,
\end{aligned}
$$

and

$$
\begin{aligned}
& \int_{0}^{\infty} k_{r}(m) \exp \left(-f_{r}(t) / \tau_{r}(m)\right) w(m) d m \\
& \quad=\int_{0}^{\infty} k_{r}(m) \exp \left(-t / \tau_{r}(m)\right) \bar{w}(m) \frac{\tau_{r}^{\prime}(m)}{\left(\tau_{r}(m)\right)^{2}} d m .
\end{aligned}
$$

The importance of the above choices for $\bar{\Phi}_{r}(m)$ and $\bar{\Psi}_{r}(m)$ is that they ensure that the following counterpart of Eq. 15 is identical to that arising from Eq. 14 when $g_{r}(t)=f_{r}(t)=t$

$$
\begin{aligned}
& \int_{0}^{\infty} \exp \left(-t / \tau_{r}(m)\right) \frac{\bar{h}_{r}(m) \tau_{r}^{\prime}(m)}{\tau_{r}(m)} d m \\
& \quad=\left[\int_{0}^{\infty} \exp \left(-t / \tau_{r}(m)\right) k_{r}(m) \bar{w}(m) d m\right]^{r} .
\end{aligned}
$$

It is these three relationships, Eqs. 16, 17, and 18, which will play the key role in the subsequent deliberations. In the situation where $g_{r}(t)=f_{r}(t)=t$, it follows that $\bar{h}_{r}(m)=h_{r}(m)$ and $\bar{w}(m)=w(m)$ and the core Eq. 18 recover this particular situation.

On the basis of these three equations, the solution of Eq. 14 to recover $w(m)$ from measurements of $h_{r}(m)$ separates naturally into the follow three steps.

Step 1. Determining $\bar{h}_{r}(m)$

On setting $p=1 / \tau_{r}(m)$ and writing $m=\phi_{r}(p)$ for its inverse, Eq. 16 becomes

$$
\begin{aligned}
& \int_{0}^{\infty} \exp \left(-g_{r}(t) / \tau_{r}(m)\right) \frac{h_{r}(m) \tau_{r}^{\prime}(m)}{\tau_{r}(m)} d m \\
& =\int_{0}^{\infty} \exp \left(-t / \tau_{r}(m)\right) \frac{\bar{h}_{r}(m)\left(\tau_{r}^{\prime}(m)\right)^{2}}{\left(\tau_{r}(m)\right)^{3}} d m \\
& =\int_{o}^{\infty} \exp (-t p) p \tau_{r}\left(\phi_{r}(p)\right) \hat{h}_{r}(p) d p \\
& =\mathcal{L}\left(p \tau_{r}\left(\phi_{r}(p)\right) \hat{h}_{r}(p)\right),
\end{aligned}
$$


where $\hat{h}_{r}(p)=\bar{h}_{r}\left(\phi_{r}(p)\right)$ and $\mathcal{L}$ denotes the Laplace transform operator

$\mathcal{L}[f(p)](s)=\int_{0}^{\infty} \exp (-s p) f(p) d p$.

If it is assumed that $g_{r}(t), h_{r}(m)$, and $\tau_{r}(m)$ are known, then the left-hand side of Eq. 19 defines the value of the Laplace transform on the right-hand side. On taking the inverse Laplace of this equation, the value of $\hat{h}_{r}(p)$ can thereby be determined. The value required of $\bar{h}_{r}(m)$ is then recovered as $\hat{h}_{r}\left(1 / \tau_{r}(m)\right)$.

On the other hand, if it is assumed that $h_{r}(m), \bar{h}_{r}(m)$, and $\tau_{r}(m)$ are known (by measuring $h_{r}(m)$ for a polymer with a known MWD $w(m)$ and assuming that $f_{r}(t)$ is known), then Eq. 19 can be solved to determine $g_{r}(t)$.

Note The importance of step 1 is that it establishes how, for any choice of $g_{r}(t)$, such as the stretched exponential function $\exp \left(-t^{\alpha}\right), 0<\alpha<1$, the corresponding relaxation modulus $G_{r}(t)$ of Eq. 12 and the RTS-MWD relationship Eq. 14 can be transformed to yield the canonical Eq. 18. The clear advantage of this approach is that, once one has a program that solves Eq. 18, the solution of Eq. 14 for different choices for $g_{r}(t)$ reduces to solving the appropriate form of Eq. 19 to determine the equivalent form for Eq. 18.

Step 2. The solution of the core Eq. 18

Using the same substitution as in step 1, Eq. 18 can be rewritten as

$$
\int_{0}^{\infty} \exp (-t p) \frac{\hat{h}_{r}(p)}{p} d p=\left[\int_{0}^{\infty} \frac{\hat{k}_{r}(p)}{p^{2} \hat{\tau}_{r}^{\prime}(p)} \exp (-t p) \hat{w}(p) d p\right]^{r},
$$

where $\hat{h}_{r}(p)=\bar{h}_{r}\left(\phi_{r}(p)\right), \quad \hat{k}_{r}(p)=k_{r}\left(\phi_{r}(p)\right), \quad \hat{\tau}_{r}^{\prime}(p)=$ $\tau_{r}^{\prime}\left(\phi_{r}(p)\right)$, and $\hat{w}(p)=\bar{w}\left(\phi_{r}(p)\right)$. This is the crucial step that leads to the construction of an exact solution because both the above integrals can be interpreted as Laplace transforms. Using as the above definition of the Laplace transform, the following equivalent expression for Eq. 21 is immediate

$\mathcal{L}\left(\frac{\hat{h}_{r}(p)}{p}\right)=\left[\mathcal{L}\left(\frac{\hat{k}_{r}(p)}{p^{2} \hat{\tau}_{r}^{\prime}(p)} \hat{w}(p)\right)\right]^{r}$.

For given $\hat{w}(p)$, the corresponding value of $\hat{h}_{r}(p)$ is given by

$\hat{h}_{r}(p)=p \mathcal{L}^{-1}\left\{\left[\mathcal{L}\left(\frac{\hat{k}_{r}(p)}{p^{2} \hat{\tau}_{r}^{\prime}(p)} \hat{w}(p)\right)\right]^{r}\right\}$ while, for given $\hat{h}_{r}(p)$, the corresponding value of $\hat{w}(p)$ is given by

$\hat{w}(p)=\frac{p^{2} \hat{\tau}_{r}^{\prime}(p)}{\hat{k}_{r}(p)} \mathcal{L}^{-1}\left(\left[\mathcal{L}\left(\frac{\hat{h}_{r}(p)}{p}\right)\right]^{1 / r}\right)$,

where $\mathcal{L}^{-1}$ denotes the inverse Laplace transform.

Note This highlights the advantage of transforming back to the canonical form of Eq. 18, as the above analysis shows how its solution can be written down in terms of Laplace transforms. In some situations, the Laplace transform relationships Eqs. 22 and 23 can be solved analytically, as shown in Section Application: the double reptation RTS-MWD relationship. More often, it will be necessary to solve them numerically.

Step 3. The determination of $w(m)$.

Using the same substitution as in step 1, the linking of the right-hand side of Eq. 14 to that of Eq. 15 can be accomplished in the following manner:

$$
\begin{aligned}
\int_{0}^{\infty} k_{r}(m) \exp \left(-f_{r}(t) / \tau_{r}(m)\right) w(m) d m \\
=\int_{0}^{\infty} k_{r}(m) \exp \left(-t / \tau_{r}(m)\right) \bar{w}(m) d m \\
=\mathcal{L}\left(\frac{\hat{k}_{r}(p)}{p^{2} \hat{\tau}_{r}^{\prime}(p)} \hat{w}(p)\right),
\end{aligned}
$$

where $\hat{w}(p)=\bar{w}\left(\phi_{r}(p)\right)$.

If it is assumed now that $k_{r}(m), \tau_{r}(m)$, and $\hat{w}(m)$ are known, then the right-hand side of this last equation can be evaluated. For a given function $f_{r}(m)$, the required value of $w(m)$ is then recovered by solving the resulting first kind Fredholm integral equation. This then defines the procedure that must be followed for each $r$ component that is hypothesized to be in the model $G_{M}(t)$ of $G(t)$ when the full generality of Eq. 17 applies. In the sequel, because of their rheological importance, attention will mainly focus on single and double reptation.

\section{Laplace transform and convolutional RTS-MWD relationships}

Henceforth, it will be assumed that $f_{r}(t)=g_{r}(t)=t$ for all chosen values of $r$. As explained in Section The general strategy for MWD recovery from the general RTS-MWD relationship, though the general formulation is given by Eq. 14, the first step is to transform it to the core Eq. 18 and solve that equation. In this way, the resulting analysis is simpler and more illustrative 
than for the general methodology outlined in Section The general RTS-MWD relationship. With respect to the above assumption, the relationship Eq. 18 takes the following form:

$$
\begin{aligned}
& \int_{0}^{\infty} \exp \left(-t / \tau_{r}(m)\right) \frac{h_{r}(m) \tau_{r}^{\prime}(m)}{\tau_{r}(m)} d m \\
& \quad=\left[\int_{0}^{\infty} \exp \left(-t / \tau_{r}(m)\right) k_{r}(m) w(m) d m\right]^{r} .
\end{aligned}
$$

Single reptation

When single reptation is the only dynamic occurring, it follows from Eq. 11 that $N=r=1$ and $W_{1}=1$, for which Eq. 25 yields

$$
\begin{gathered}
\int_{0}^{\infty} \exp \left(-t / \tau_{1}(m)\right)\left[\frac{h_{1}(m) \tau_{1}^{\prime}(m)}{\tau_{1}(m)}-k_{1}(m)\right. \\
\left.\quad \times \exp \left(-t / \tau_{1}(m)\right) w(m)\right] d m=0 .
\end{gathered}
$$

Using the argument given in Anderssen and Loy (2002a), this last equation solves to yield

$w_{1}(m)=\frac{h_{1}(m) \tau_{1}^{\prime}(m)}{k_{1}(m) \tau_{1}(m)}$.

Note In fact, it follows from the deliberations in Anderssen and Loy (2002a) that, when $r=1$, Eq. 14 has a unique solution for given choices for $f_{1}$ and $g_{1}$ and, in addition, Eq. 27 defines that solution when $f_{1}=g_{1}$.

With $k_{1}(m)=1 / m$ and $\tau_{1}(m)=k m^{\alpha}$, this last relationship becomes

$w_{1}(m)=\alpha h_{1}(m)$,

which implies that $w_{1}(m)$, up to a multiplicative constant that gives the correct units, simply corresponds to a molecular weight scaling of the RTS $H_{1}(\tau)$, associated with the measured $G_{1}(t)$, to generate $h_{1}(m)$. This result was given in Thimm et al. (1999) and corresponds to setting $\beta=1$ in Eqs. 7 and 8 above. The elementary nature of this result is a direct consequence of the assumptions that $r=1$, and $f_{1}(t)=g_{1}(t)$.

However, experimental results show that this is too simple a relationship because it fails to perform the sufficiently strong sharpening of $H_{1}(\tau)$ required to recover $w_{1}(m)$ as a delta function when $G_{1}(t)$ corresponds to the measured relaxation modulus of a monodisperse polymer. Consequently, either single reptation in the above form is too simple a model for MWD recovery, or it holds, but the classical assumption $f_{1}(t)=$ $g_{1}(t)=t$ does not. This represents a possible role for the Kohlrausch functions $\exp \left(-t^{\alpha}\right), 0<\alpha<1$. As explained in Anderssen and Loy (2002a), depending on the nature of $f_{1}(t)$ and $g_{1}(t)$, Eq. 14 can have a unique solution.

\section{Double reptation}

When double reptation is the only dynamics, it follows from Eq. 11 that $N=2, W_{1}=0$, and $W_{2}=1$, for which Eq. 25 yields

$$
\begin{aligned}
& \int_{0}^{\infty} \exp \left(-t / \tau_{2}(m)\right) \frac{h_{2}(m) \tau_{2}^{\prime}(m)}{\tau_{2}(m)} d m \\
& \quad=\left[\int_{0}^{\infty} k_{2}(m) \exp \left(-t / \tau_{2}(m)\right) w_{2}(m) d m\right]^{2} .
\end{aligned}
$$

Now, however, the logic that allowed one to go from Eq. 26 to Eq. 27 cannot be applied. It is therefore necessary, as in step 2 of Section The general RTS-MWD relationship, to transform Eq. 29 to its equivalent Laplace transform counterpart; namely,

$\int_{0}^{\infty} \exp (-t p) \frac{\hat{h}_{2}(p)}{p} d p=\left[\int_{0}^{\infty} \frac{\hat{k}_{2}(p)}{p^{2} \hat{\tau}_{2}^{\prime}(p)} \exp (-t p) \hat{w}(p) d p\right]^{2}$,

where $\hat{h}_{2}(p)=h_{2}\left(\phi_{r}(p)\right), \hat{k}_{2}(p)=k_{2}\left(\phi_{2}(p)\right), \hat{\tau}_{2}^{\prime}(p)=$ $\tau_{2}^{\prime}\left(\phi_{2}(p)\right)$, and $\hat{w}(p)=w\left(\phi_{r}(p)\right)$.

The utilization of this result can be performed in two different ways.

\section{The Laplace transform solution}

Equation 30 can be written

$\mathcal{L}\left(\frac{\hat{h}_{r}(p)}{p}\right)=\left[\mathcal{L}\left(\frac{\hat{k}_{r}(p)}{p^{2} \hat{\tau}_{r}^{\prime}(p)} \hat{w}(p)\right)\right]^{2}$.

For given $\hat{w}(p)$, the corresponding value of $\hat{h}_{2}(p)$ is given by

$\hat{h}_{2}(p)=p \mathcal{L}^{-1}\left\{\left[\mathcal{L}\left(\frac{\hat{k}_{2}(p)}{p^{2} \hat{\tau}_{2}^{\prime}(p)} \hat{w}(p)\right)\right]^{2}\right\}$

while, for given $\hat{h}_{2}(p)$, the corresponding value of $\hat{w}(p)$ is given by

$\hat{w}(p)=\frac{p^{2} \hat{\tau}_{2}^{\prime}(p)}{\hat{k}_{2}(p)} \mathcal{L}^{-1}\left[\mathcal{L}\left(\frac{\hat{h}_{2}(p)}{p}\right)\right]^{1 / 2}$. 


\section{The Laplace convolution theorem solution}

Applying the Laplace convolution theorem to the righthand side of Eq. 30 gives

$$
\begin{aligned}
\int_{0}^{\infty} \exp (-t p) \frac{\hat{h}_{2}(p)}{p} d p \\
=\left[\int_{0}^{\infty} \exp (-t p)\right. \\
\left.\quad \times \int_{0}^{p} F(q, p-q) \hat{w}_{2}(q) \hat{w}_{2}(p-q) d q\right] d p,
\end{aligned}
$$

where

$F(q, p-q)=\frac{\hat{k}_{2}(q) \hat{k}_{2}(p-q)}{q^{2}(p-q)^{2} \hat{\tau}_{2}^{\prime}(q) \hat{\tau}_{2}^{\prime}(p-q)}$.

The uniqueness of the Laplace transform then yields

$\frac{\hat{h}_{2}(p)}{p}=\int_{0}^{p} F(q, p-q) \hat{w}_{2}(q) \hat{w}_{2}(p-q) d q$.

The importance and interesting aspect of this relationship is the explicit characterization it gives of the polymer dynamics occurring in double reptation. It shows how the RTS depends on the convolution of the (transformed) MWD (of the polymer molecules) with the convolution under the influence of a kernel function that characterizes how the relaxation times depend on the molecular weights. In addition, the convolutional process naturally allows for all possible cross-linking to occur between the various polymer molecules that make up their MWD.

\section{Application: the double reptation RTS-MWD relationship}

The Laplace transform solution of Eq. 30 can now be utilized to give an analytic illustration of the relationship between the RTS and MWD peaks. Let the RTS $H(\tau)$ be given by

$H(\tau)=\frac{1}{(2 n) !}\left(\frac{1}{\tau}\right)^{2 n} \exp (-\beta / \tau)$,

where it is assumed that $G_{0}^{N}=1$, and the normalization condition

$\int_{0}^{\infty} \frac{H(\tau)}{\tau} d \tau=1$

which yields the constraint

$\beta=(2 n)^{-(1 /(2 n))}$.
Assuming that double reptation is the only polymer dynamics active, it follows, for the appropriate choice for $\tau=\tau_{2}(m)$, that

$h_{2}(m)=H\left(\tau_{2}(m)\right)=\frac{1}{(2 n) !}\left(\frac{1}{\tau_{2}(m)}\right)^{2 n} \exp \left(-\beta / \tau_{2}(m)\right)$.

Thus, on performing the substitution $p=1 / \tau_{2}(m)$, this last equation becomes

$\hat{h}_{2}(p)=\frac{1}{(2 n) !} p^{2 n} \exp (-\beta p)$.

From Eq. 33, using the fact that

$\mathcal{L}\left[\frac{p^{2 n-1}}{(2 n-1) !} \exp (-\beta p)\right]=\left(\frac{1}{s+\alpha}\right)^{2 n}$,

it follows, after standard algebraic manipulations, that

$\hat{w}_{2}(p)=\frac{\hat{\tau}_{2}^{\prime}(p)}{\hat{k}_{2}(p)} \frac{1}{\sqrt{2 n}} \frac{p^{n+1}}{(n-1) !} \exp (-\beta p)$,

and, hence,

$w_{2}(m)=\frac{\tau_{2}^{\prime}(m)}{k_{2}(m)} \frac{1}{\sqrt{2 n}} \frac{\exp \left(-\beta / \tau_{2}(m)\right)}{(n-1) !\left(\tau_{2}(m)\right)^{n+1}}$.

Alternatively, if one starts with $w_{2}(m)$ in the form Eq. 41, then the corresponding $H(\tau)$ is given by Eq. 37 .

Both $h_{2}(m)$ and $w_{2}(m)$ are unimodal peaks and, therefore, represent an appropriate framework in which to explore the interdependence between $h_{2}(m)$ and $w_{2}(m)$ when the MWD has a unimodal structure, which is often the situation in polymer rheology.

Substitution of $\tau_{2}(m)=\mathrm{km}^{\alpha}$ in the above relationships yields

$h_{2}(m)=\frac{1}{(2 n) !\left(k m^{\alpha}\right)^{2 n}} \exp \left(-\beta /\left(k m^{\alpha}\right)\right)$

and, assuming that $k_{2}(m)=1 / m$,

$w_{2}(m)=\frac{\alpha}{\sqrt{2 n}(n-1) !\left(k m^{\alpha}\right)^{n}} \exp \left(-\beta /\left(k m^{\alpha}\right)\right)$.

Consequently, the unimodal $h_{2}(m)$ peaks at $m=$ $[\beta /(2 k n)]^{1 / \alpha}$, which is always to the left of the peak of the unimodal $w_{2}(m)$ at $m=[\beta /(k n)]^{1 / \alpha}$. This could be an artifact of the above choice of model, in which it is assumed that $k_{2}(m)=1 / m$, but it could also be saying something deep about the interdependence of $h_{2}(m)$ and $w_{2}(m)$ in situations where something like the above assumptions apply. 


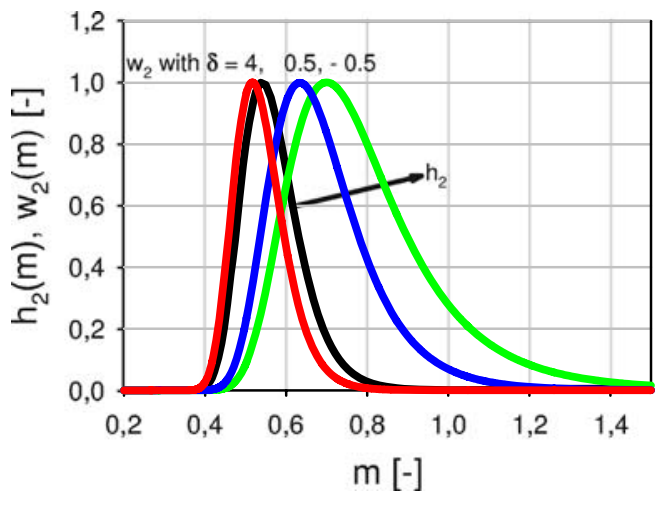

Fig. 2 Graphical comparisons of $h_{2}(m)$ (solid line) with the corresponding $w_{2}(m)$ of Eq. 42, for various choices of the parameters $[k=1, n=3, \alpha=3.4, \delta=-0.5$ (dots), 0.5 (dashes on right), 4 (dashes on left)] illustrating the interdependence between these two functions

Interestingly, and quite importantly, a small change in the way that $k_{2}(m)$ is modelled can cause a major change in the interrelationship between $h_{2}(m)$ and $w_{2}(m)$. It is a direct illustration of the fact that the inherent improperly posedness is there at the analytic level. For example, on choosing $k_{2}(m)=1 / m^{1-\delta \alpha}$ for some $\delta>0$, one obtains

$w_{2}(m)=\frac{\alpha k^{\delta}}{\sqrt{2 n}(n-1) !\left(k m^{\alpha}\right)^{n+\delta}} \exp \left(-\beta /\left(k m^{\alpha}\right)\right)$,

which peaks at $m=\left(\beta /((n+\delta) k)^{1 / \alpha}\right.$. Consequently, for $\delta>n, w_{2}(m)$ will peak to the left of $h_{2}(m)$.

Graphical comparisons of $h_{2}(m)$ with the corresponding $w_{2}(m)$ yield a clear illustration of how the RTS peak is a modification and relocation of the MWD peak, which is consistent with experimental observations of their interdependence. This is illustrated in Fig. 2, where the normalization condition $\beta=$ $(2 n)^{-1 /(2 n)}$ has been applied, along with appropriate scaling so that the peaks of $h_{2}(m)$ and $w_{2}(m)$ all have the same height. They show clearly that, even for these simple analytic models, there can be great variability in the shape and positioning of $w(m)$ relative to the observed shape and positioning of $h_{2}(m)$, and vice versa.

It raises the interesting question of whether, for experimentally measured $h_{2}(m)$ for given monodisperse $w_{2}(m)$, the position of the peak of $w_{2}(m)$ is consistently on one side or the other of the peak of $h_{2}(m)$, or whether it tends to change from one side to the other depending on the type of polymer being examined. Consequently, one obtains direct confirmation of the need for rigorous solutions, for RTS-MWD relationships, along the lines outlined in this paper.

\section{Rheological implications illustrated with the BSW model}

The motivation behind the need to put the mathematical modelling of RTS-MWD relationships on a rigorous mathematical footing has been given in Sections Introduction and Rheological background. The recent paper of Nobile and Cocchini (2008) represents addition validation for this point of view. Here, in this section, the aim is to give a brief overview of the practical rheological situation. Because of its popularity, the BSW model Baumgartel et al. (1990) has been chosen as the model for the relaxation spectrum $H(\tau)$.

It is known that the BSW model of the relaxation spectrum $H(\tau)$ (Baumgartel et al. 1990) and related models (Friedrich et al. 2008) yield good approximations to the measured dynamic modulii $G^{\prime}(\omega)$ and $G^{\prime \prime}(\omega)$ of long, linear, and flexible polymers. Here, this will be illustrated for the PS664 polystyrene, which has a molecular weight of $\mathrm{Mw}=634,700 \mathrm{~g} / \mathrm{mol}$ and a polydispersity index of $\mathrm{Mw} / \mathrm{Mn}=1.07$. Values of the dynamic modulii for this polymer are plotted in Fig. 3, where the circles and boxes correspond to the measured values of $G^{\prime}(\omega)$ and $G^{\prime \prime}(\omega)$, respectively, while the solid and dashed lines correspond to the values of $G^{\prime}(\omega)$ and $G^{\prime \prime}(\omega)$ generated by specific choices for the parameters in the modified BSW model

$H_{\mathrm{BSW}}(\tau)=n_{e} G_{c} \frac{x^{n_{e}}}{\left(1+x^{\beta}\right)^{1+n_{e} / \beta}}, \quad x=\tau / \tau_{c}$,

where $x$ corresponds to the ratio of the relaxation time $\tau$ and a characteristic relaxation time $\tau_{c} ; G_{c}$ a characteristic (plateau) modulus; $n_{e}$, as for the simple BSW

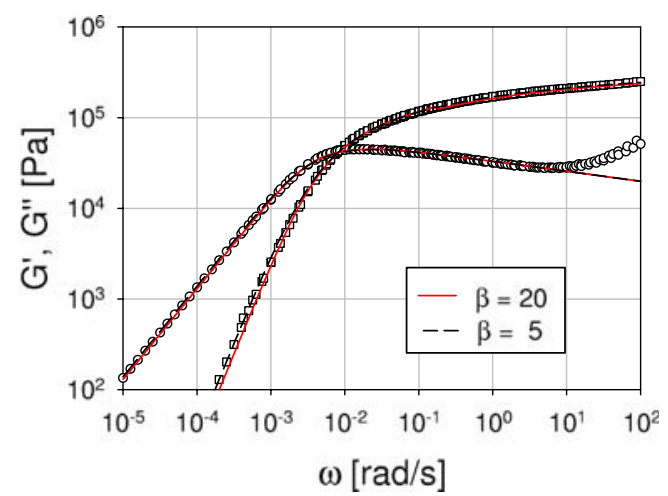

Fig. 3 Dynamic modulii $G^{\prime}$ (blue dots) and $G^{\prime \prime}$ (red boxes) for the polymer PS644 together with the predictions for the generalized BSW spectrum [dashed line for $\beta=20$ (which corresponds effectively to the original BSW behavior with a discontinuous cut-off) and solid line for $\beta=5$ ] 


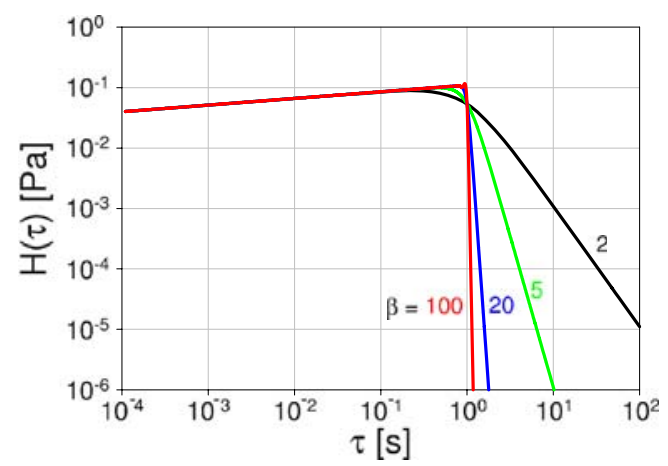

Fig. 4 The BSW spectrum, corresponding to Eq. 43, with $n_{e}=$ $0.11, G_{c}=360,000 \mathrm{~Pa}, \tau_{c}=1 \mathrm{~s}$, and $\beta=100,20,5,2$ (from left to right)

model, the slope of $\log \left(G^{\prime \prime}(\log \omega)\right)$ in the entanglement region; and $\beta$ the parameter describing the cut-off in relaxation times greater than $\tau_{c}$. The original BSW model is recovered as $\beta \rightarrow \infty$.

The values of $G^{\prime}(\omega)$ and $G^{\prime \prime}(\omega)$, generated when $n_{e}=$ $0.11, G_{c}=360,000 \mathrm{~Pa}, \tau_{c}=360$ in Eq. 43 , are plotted for $\beta=5$ and $\beta=20$ in Fig. 3. The very good agreement between them and the data confirm the excellent predictive power of the modified BSW spectrum for long, linear, and flexible polymers. However, a closer comparison of the plots for $G^{\prime}(\omega)$ and $G^{\prime \prime}(\omega)$, when $\beta=5$ and $\beta=20$, indicates that the $\beta=5$ results give a better fit to the data in the terminal zone. Consequently, it was deemed not necessary to examine the situation for values of $\beta$ higher than 20 .

The role of $\beta$ is illustrated in Fig. 4. It corresponds to the slope of the modified BSW model for $x>1$. With $\beta=20$, the modified model is already very close to the original $\mathrm{BSW}$ model, while, for $\beta=100$, the modified

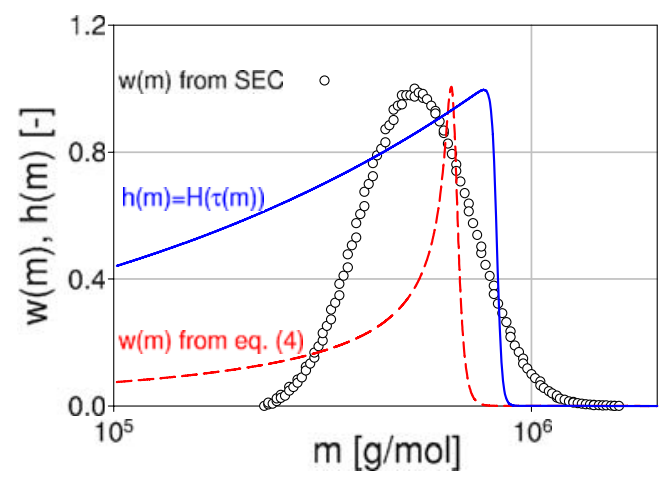

Fig. 5 A comparison of the MWD for the polymer PS644 (circles) with the MWD derived from Eq. 4 for the BSW spectrum with $\beta=20$ (solid line) and the corresponding relaxation time spectrum as a function of the molecular weight $m$ (dashed line) model reproduces the original BSW model to graphical accuracy.

Interestingly, even though there is a clear difference in Fig. 4 between the plots of $H_{\mathrm{BSW}}(\tau)$ when $\beta=5$ and $\beta=20$, the corresponding differences between $G^{\prime}(\omega)$ and $G^{\prime \prime}(\omega)$ are quite minor. This is an illustration of the underlying improperly posedness, where only small changes in $G^{\prime}(\omega)$ and $G^{\prime \prime}(\omega)$ can correspond to large changes in the relaxations spectrum and, thereby, the reason why the recovery of estimates of $H(\tau)$ from measurements of $G^{\prime}(\omega)$ and $G^{\prime \prime}(\omega)$ is a challenging problem.

The independently measured MWD for PS664 is shown as the plot of circles in Fig. 5 as first published in Maier et al. (1998).

The resulting MWD, obtained by substituting the above BSW model with $\beta=20$ into the formula proposed by Wu (1988) (Eq. 4 in the main text), is plotted in Fig. 5 along with this BSW model. Here, the relaxation time dependence on the molecular weight $m$ was assumed to take the standard form $\tau(m)=k\left(m / m_{u}\right)$ with the Maier et al. (1998) values of $k=6.92 \times$ $10^{-20} s, m_{u}=1 \mathrm{~g} / \mathrm{mol}, \alpha=3.67$. A comparison of the independently measured MWD (the circles) with the MWD reconstructed by using the mentioned BSW model indicates that the resulting reconstructed MWD yields an unacceptable approximation to the measured MWD.

The same comparison is shown in Fig. 1 for the above BSW model with $\beta=5$, which gives a better fit to the measured dynamic modulii. The reconstructed MWD now yields a better approximation to the measured MWD. Though there has only been a small change in the BSW model substituted into Eq. 4, the corresponding change in reconstructed MWD has been quite large. This illustrates another aspect of the improperly posedness associated with the recovery of an MWD from rheological measurements. A comparison of the recovered MWD in Fig. 1 with that in Fig. 5 implies that the cut-off in the original BSW model is too sharp in not allowing for the relaxation times to smoothly (as well as quickly) approach zero.

Different reasons can be given as to why the recovery of the MWD through the use of the modified BSW model (with $\beta=5$ ) and the formula of $\mathrm{Wu}$ (1988) fail to give a realistic approximation to the independently measured MWD. They must not only include shortcomings in the modified BSW model, in terms of the way it approximates the internal relaxations in the linear chains, but also the shortcomings, discussed above, in the current formulas being used to perform the reconstruction of the MWD from measurements of the dynamic modulii. 
Acknowledgements The last author wishes to acknowledge support from the Johann Radon Institute for Computational and Applied Mathematics, Austrian Academy Sciences, and the Freiburg Materials Research Center (FMF), University of Freiburg, which allowed him to progress this research while on a visit to Europe. Thanks are also due to the comments of the referees, which resulted in an improved paper.

\section{References}

Anderssen RS, Loy RJ (2002a) Completely monotone fading memory relaxation modulii. Bull Aust Math Soc 65:449-460

Anderssen RS, Loy RJ (2002b) Rheological implications of completely monotone fading memory. J Rheol 46:1459-1472

Anderssen RS, Husain SA, Loy RJ (2004) Kohlrausch functions: properties and applications. ANZIAM J (E) 45:C800-C816

Bernstein SN (1928) Sur les fonctions absolument monotones. Acta Math 52:1-66

Baumgartel M, Schausberger A, Winter HH (1990) The relaxation of polymers with linear flexible chains of uniform length. Rheol Acta 29:400-408

Cocchini F, Nobile MR (2003) Constrained inversion of rheological data to molecular weight distribution for polymer melts. Rheol Acta 42:232-242

Doi M (1981) Explanation for the 3.4 power law of viscosity of polymeric liquids on the basis of the tube model. J Polym Sci Part C Polym Lett 19:265-273

Ferry J (1980) Viscoelastic properties of polymers. Wiley, New York

Friedrich C, Waizenegger W, Winter HH (2008) Relaxation patterns of long linear flexible monodisperse polymers: BSE spectrum revisited. Rheol Acta 47:909-916

Garcia-Franco CA, Mead DW (1999) Rheological and molecular characterization of linear backbone flexible polymers with the Cole-Cole model relaxation spectrum. Rheol Acta 38: $34-47$

Husain SA, Anderssen RS (2005) Modelling the relaxation modulus of linear viscoelasticity using a Kohlrausch function. JNNFM 125:157-170

Macdonald JR (2000) On relaxation-spectrum estimation for decades of data: accuracy and sampling localization considerations. Inverse Probl 16:1561-1583

Maier D, Eckstein A, Friedrich C, Honerkamp J (1998) Evaluation of models combining rheological data with molecular weight distributions. J Rheol 42:1153-1173

Milner ST, McLeish TCB (1998) Reptation and contour-length fluctuations in melts of linear polymers. Phys Rev Lett $81: 725-728$

Nobile MR, Cocchini F (2000) Predictions of linear viscoelastic properties for polydisperse entangled polymers. Rheol Acta 39:152-162

Nobile MR, Cocchini F (2001) Evaluation of molecular weight distribution from dynamic moduli. Rheol Acta 40:111-119

Nobile MR, Cocchini F (2008) A general relation between MWD and relaxation time spectrum. Rheol Acta 47:509-519

Thimm W, Friedrich C, Marth M, Honerkamp J (1999) An analytical relation beteen relaxation time spectrum and molecular weight distribution. J Rheol 43:1663-1672

Thimm W, Friedrich C, Marth M, Honerkamp J (2000) On the Rouse spectrum and the determination of the molecular weight distribution from rheological data. J Rheol 44: 429-438

van Ruymbecke E, Keunings R, Stephenne V, Hagenaars A, Bailey C (2002) Evaluation of reptation models for predicting the linear viscoelastic properties of entangled linear polymers. Macromolecules 35:2689-2699

Widder DV (1972) The laplace transform. Princeton University Press, Princeton

Wu S (1988) Characterization of polymer molecular weight distribution by transient viscoelasticity: polytetrafluoroethylenes. Polym Eng Sci 28:538-543 